Revue scientifique francophone en Communication

organisationnelle

$18 \mid 2000$

Non-verbal, communication, organisation

\title{
La « sur vision » comme inducteur de création de
} sens

Jean-Luc Michel

\section{OpenEdition}

\section{Journals}

Édition électronique

URL : https://journals.openedition.org/communicationorganisation/2420

DOI : 10.4000/communicationorganisation.2420

ISSN : 1775-3546

\section{Éditeur}

Presses universitaires de Bordeaux

\section{Édition imprimée}

Date de publication : 1 novembre 2000

ISSN : 1168-5549

Référence électronique

Jean-Luc Michel, «La «sur vision » comme inducteur de création de sens », Communication et organisation [En ligne], 18 | 2000, mis en ligne le 27 mars 2012, consulté le 05 août 2021. URL : http:// journals.openedition.org/communicationorganisation/2420; DOI : https://doi.org/10.4000/ communicationorganisation. 2420

Ce document a été généré automatiquement le 5 août 2021 .

(C) Presses universitaires de Bordeaux 


\title{
La « sur vision » comme inducteur de création de sens
}

\author{
Jean-Luc Michel
}

Dans le précédent colloque consacré à "L'induction dans la communication", le rapport au non-verbal était continuellement sous-jacent. Il s'agit à présent d'essayer de comprendre comment le sens peut-il «passer » en dehors ou en marge des processus langagiers. La recherche dont cet article rend compte s'est centrée sur les structures visuelles en les restituant dans une perspective interactive et multidimensionnelle. Après avoir établi que le concept d'induction était entièrement «décrit " par des successions de phases de distanciation et d'identification, notre contribution de 1997 proposait d'élargir la réflexion en croisant des approches cognitives et esthétiques : la conclusion affirmait que "l'induction pourrait être définie comme un transfert d'excitation de réseaux sémantiques ». C'est dans cet axe que des travaux déjà anciens sur le concept de " sur vision » ont été repris, actualisés et élargis, notamment à l'aune des applications hyper-médiatiques les plus récentes. Cet article présente une brève genèse du concept, montre l'élargissement à faire subir à la notion centrale d'information, et après quelques exemples de mise en œuvre de méthodes pour «sur voir », il élargit la recherche à ce qui peut être dénommé une « pensée graphique » ou un « inducteur polysensoriel » pour déboucher sur une proposition d'élargissement, de « dépassement » de la transmission des contenus sémantiques.

\section{De l'induction à la sur vision, une permanence de la distanciation}

Dans notre contribution au colloque précité, la sur vision fut rapidement présentée comme exemple en action de nos capacités inductives, de nos profils (inducteurs "distanciateurs » et "identificateurs» ou " projectifs ", etc. ${ }^{1}$. L'idée centrale qui se trouve derrière ces notions est naturellement la « théorie » de la distanciation que nous essayons de construire depuis plusieurs années ${ }^{2}$ - Cette approche - radicalement non déterministe - stipule que l'être humain oscille sans arrêt entre une attitude 
"distanciatrice» et une attitude «identificatrice»; sa liberté et son unicité ontologiques résident essentiellement dans cette alternance qui rend compte en même temps de toutes les situations de communication. En 1997. nous avions montré que l'identification était le moteur de l'induction et que la distanciation en était le catalyseur. Cet aboutissement constitue le point de départ de la recherche exposée ici. La perspective interactionniste qu'offre la problématique du colloque permet de recadrer le non-verbal dans l'induction. Que ce soient la «représentation de soi » goffmanienne, la "méta-communication" batesonienne, voire l'intentionnalité de Searle (ou de Merleau-Ponty), on trouve facilement des traces ou des marqueurs non verbaux plus ou moins inducteurs. Le non-verbal apporte sa part de complexité (certains diraient de dualité) aux théories de la communication, et ce n'est pas sa moindre qualité. Mais il charrie aussi un risque majeur qui ne sera que signalé ici parce ce qu'il borne nos travaux: par "non-verbal». on entend trop souvent "non intentionnel » comme si une sorte de lien tacite existait entre ces deux notions. Notre cadre théorique général et les travaux ayant préludé à la présente contribution montrent qu'il n'en est rien: le non-verbal peut être délibérément intentionnel, l'induction le démontre sans conteste. Mais alors qu'en est-il du non-verbal non intentionnel, du non-verbal qui parasiterait la «bonne » communication? Moles dans Théorie de l'information et perception esthétique a posé des jalons : sous forme lapidaire, on pourrait dire que le bruit fait sens, le parasite apporte lui aussi de l'information tout comme l'absence d'information est une information. Dès lors le non-verbal induit des indices en phase ou hors phase avec le processus en cours, il confirme ou infirme la transmission, la reconnaissance ou la recréation de sens.

3 Ce détour par l'intentionnalité fait saisir qu'entre l'induction et le non-verbal existent des relations intimes, sûrement dialectiques dont une approche distanciatrice peut rendre compte avec économie et efficacité. C'est pourquoi, avant d'examiner plus en détail la sur vision, un retour sur le concept fondamental d'information s'impose.

\section{L'information en tant que combinatoire complexe}

4 La leçon du colloque sur l'induction nous a semblé claire: on ne peut comprendre l'information et l'induction que dans la polysensorialité. L'insistance d'Hubert Montagner, une partie des contributions et nos propres travaux convergent vers une conclusion un peu péremptoire: le transfert d'information n'est jamais unidimensionnel, sauf protocole expérimental. Un an plus tard, en 1998, au terme d'une journée d'hommage qui lui était consacrée par l'IS1C, c'est Robert Escarpit qui, en revenant sur le positionnement épistémologique français auquel il avait lui même largement contribué, énonça avec toute sa malice qu'au fond : «l'information existait déjà dans l'esprit de l'interlocuteur, l'objet de la communication étant de vérifier que la transmission avait été bonne $»^{3}$

5 C'est un peu le croisement de ces deux conclusions qui nous a incité à revenir sur cet étrange concept d'information, bien peu interrogé depuis des lustres. Il n'est certes pas dans l'objet de cette contribution de le définir de manière révolutionnaire, mais depuis Léon Brillouin. bien peu de travaux «fondamentaux » ont été effectués dans les SIC au contraire d'autres disciplines comme la biologie. Cette contribution nous donne l'occasion d'exposer - sans la commenter en détail car la place manque - le résultat d'une recherche qui a tenté de rassembler l'ensemble des constituants du concept 
d'information, envisagé en continuité avec la communication, conformément au lien « inforcom » bien connu.

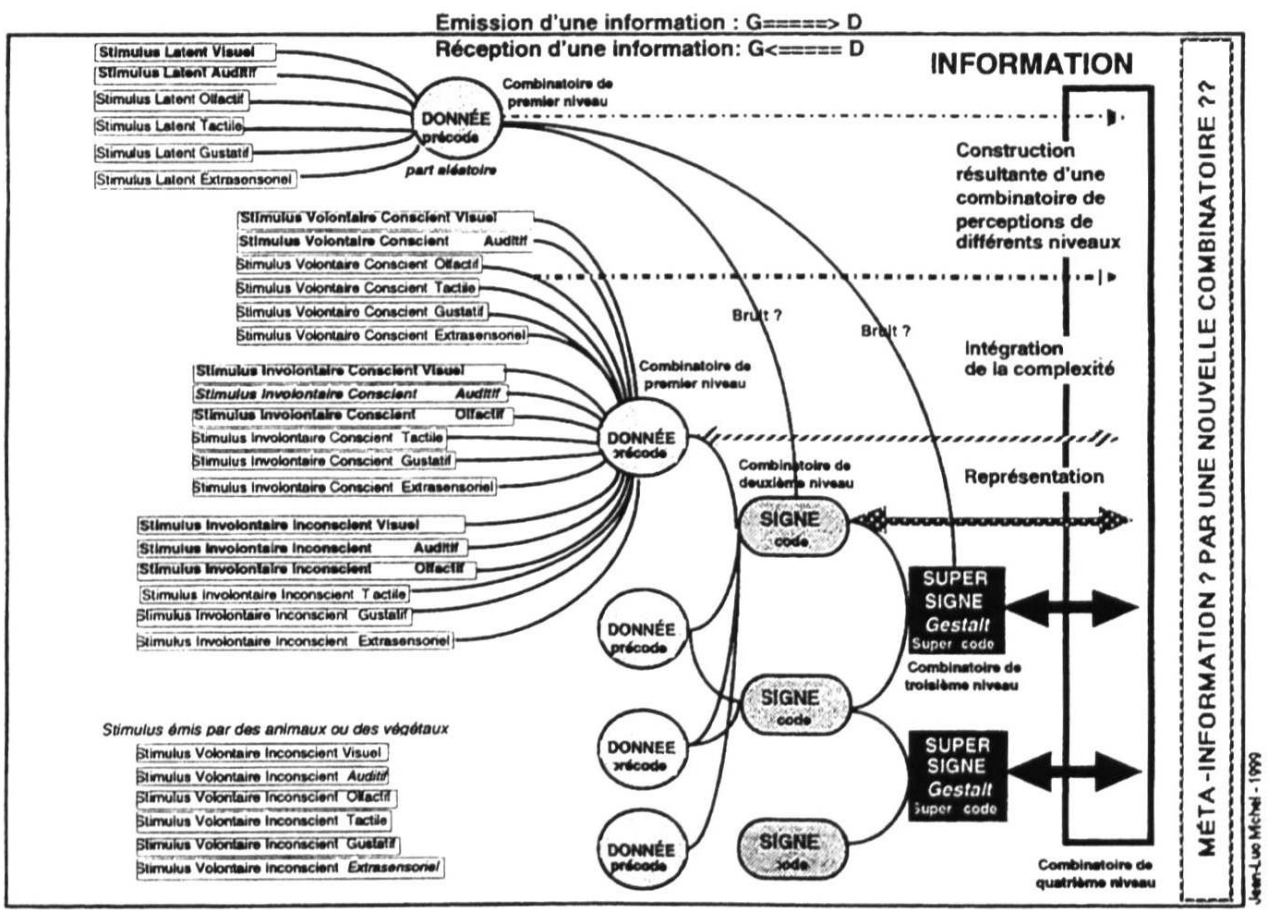

Figure 1. Les « particules élémentaires » de l'information :

6 Ce schéma essaie d'offrir une représentation synthétique de la polysensorialité de la communication. Il montre que l'information est une combinatoire complexe résultant de sous-combinatoires dans lesquelles chacun des canaux ou des sens joue son rôle ou sa "partition ». Le non-verbal se trouve replacé dans une double problématique de la complexité interactionnelle et de la dialectique de l'intentionnalité. Dans ce continuum, l'information ou la méta information sont la résultante de stimuli, de signes et de super signes, de gestalts enchevêtrés selon qu'ils sont volontaires, involontaires, conscients ou inconscients (cf. les descriptions de Goffman sur les actes volontaires ou nos propres travaux sur cette question ${ }^{4} \mathrm{La}$ " communication » serait alors tout naturellement le processus d'établissement et de gestion de la relation. Dans cette hypothèse, le non-verbal possède bien la place éminente qui doit être la sienne, en conjonction - et en interaction - directes avec le verbal ${ }^{5}$. Ces prémisses posées, il reste à voir ce qu'est la sur vision et comment elle s'établit.

\section{Genèse du concept de sur vision et exemples de mise en œuvre}

7 La sur vision est née dans la pratique de l'enseignement secondaire, en écho aux premiers emplois des «NTE» (nouvelles technologies d'enseignement). Il s'agissait au début d'utiliser celles ci pour améliorer l'acquisition de certaines notions sur lesquelles les élèves achoppaient régulièrement, par exemple en mathématiques. L'idée de départ, inchangée aujourd'hui, consistait à partir de l'observation que souvent celui qui ne comprend pas dit qu'il ne «voit» pas la solution. Ce qui peut signifier que celui qui 
trouve la voit, ou pour marquer la différence, qu'il la « survoit », d'où le terme de « sur vision " forgé pour la circonstance et que nous avions la faiblesse de croire être un néologisme jusqu'à ce que François Richaudeau nous indique un article de 1961 dans lequel un mathématicien, G. Cordonnier, l'avait employé semble-t-il le premier ${ }^{6}$ La sur vision est donc l'action de voir au delà de la stricte réalité des apparences, mais elle active des structures cognitives graphiques et conceptuelles plus profondes. C'est en ce sens que l'élève qui survoit la bonne structure mathématique, physique, biologique ou grammaticale pourra aller plus vite et plus loin. Le lecteur aura remarqué que la sur vision se rattache au concept de distanciation, en survoyant, on se distancie d'une réalité perçue, mais pour agir dessus, on identifie, à défaut de s'y identifier, ce qui fait retrouver la vision dialectique garante de la liberté ontologique signalée plus haut. Quand elle est bien rodée (ce qui signifie qu'on puisse la développer et l'entrainer ${ }^{7}$, elle devient un outil de gestion du non-verbal. Quand on survoit une structure, on découvre de l'information ou de la signification latente ou cachée. Naturellement, la sur vision n'exprime qu'un des canaux du non-verbal, mais sa diversité s'étend de la simple reconnaissance de structures graphiques formelles à la gestuelle en passant par les mimiques et autres «mises en scène de la vie quotidienne». Le mime exploite évidemment la sur vision en conduisant ses spectateurs à repérer, voir ou comprendre ce qu'il veut leur signifier, de même que les mimiques d'aides des enseignants ou des parents compatissants. Dans ces exemples, il s'agit d'exploiter des ressources nonverbales pour optimiser la communication, même s'il est des circonstances où la sur vision peut induire des sens parallèles, des connotations suggérées ou orientées, voire susciter une inventivité totalement débridée.

Les travaux développés à partir des mathématiques restent d'actualité et demeurent utilisables tels quels ou mis en scène dans des présentations assistées par ordinateur. En voici quelques aperçus :

Dans le premier cas, il s'agit simplement de montrer que derrière les produits de variables ac et ad, on peut penser à d'autre variables - ou des paramètres - par exemple $\mathrm{m}$ et $\mathrm{n}$ censés les remplacer dans des calculs. La sur vision consiste à penser $\mathrm{m}$ et $\mathrm{n}$ « au travers » de ac et ad et donc à les substituer « comme par magie ».

Figure 2. Substitution de variables (vue d'une des étapes d'un "fondu enchaîné ") :
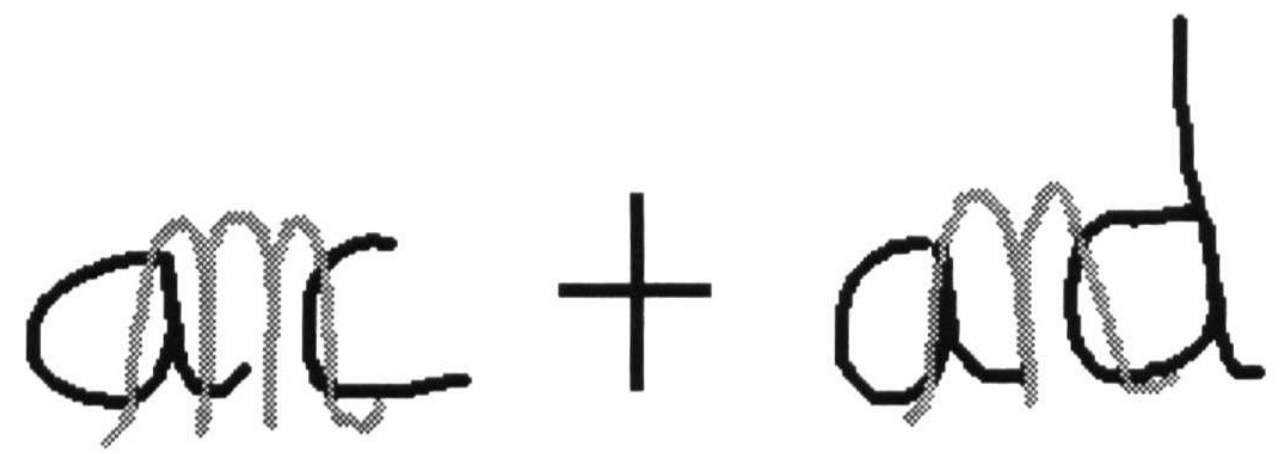

Naturellement, des variations de graphisme peuvent être employées pour conserver toute sa puissance à la sur vision et l'empêcher de sombrer dans une routine stérile. 

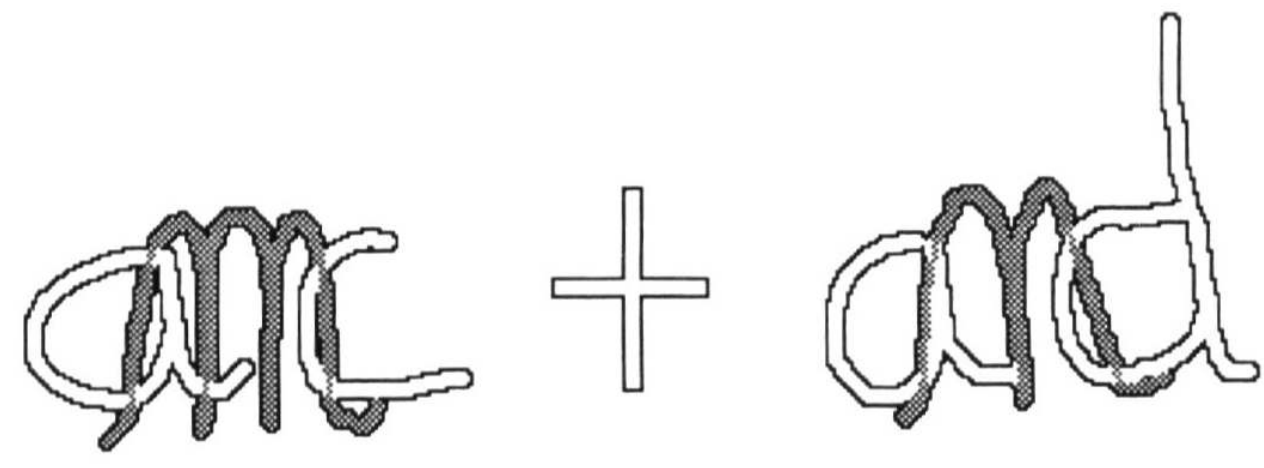

11 Au-delà de ces exemples simples et faciles à mettre en œuvre, on peut en citer beaucoup d'autres dans tous les domaines pédagogiques, de la géographie, la chimie ou l'économie à l'histoire ou aux sciences de la vie. Pendant longtemps, il ne pouvait s'agir que d'une sorte d'artisanat recourant à des subterfuges graphiques voire à du bricolage audiovisuel, alors qu'aujourd'hui, les hypermédias offrent des possibilités infiniment plus grandes. Certains films à succès, très prégnants comme Matrix (en 1999) vont même jusqu'à inscrire une sorte de sur vision au cœur du scénario: la réalité serait virtuelle et le sauvetage de l'humanité passerait par une distanciation radicale des illusions imposées par les "machines ». Il est des cas où, comme l'avait décrit de manière prémonitoire Sherry Turkle en 1988, la grande fréquentation des ordinateurs engendre une vision différente, plus distanciée, du monde en révélant un second soi même ${ }^{8}$

\section{La sur vision, inducteur polysensoriel ?}

12 L'induction non-verbale engendrée par la sur vision nécessite un cadre conceptuel plus large que l'on trouvera autour de la "pensée graphique », c'est-à-dire une pensée qui s'affranchirait de ce qu'André Leroi-Gourhan a nommé «le carcan rectilinéaire de

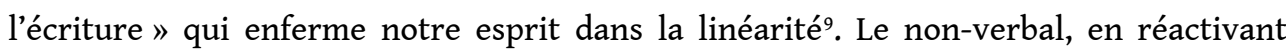
d'autres pôles contribuerait alors à nous libérer. Dès lors, la vulgate selon laquelle les réseaux nous apporteraient une liberté plus grande - si elle doit être toujours critiquée au plan social ou politique (une plus grande liberté pour qui ?) mérite néanmoins un examen plus attentif que la condescendance habituelle des chercheurs le suggérerait: la pratique des hypertextes et des hypermédias nous entraîne à la distanciation et donc en même temps à l'identification, de sorte que se construisent rapidement des " procédures mentales » où le non-verbal acquiert une importance déterminante dans la construction et la reconstruction des significations.

Dès lors, on peut inférer que la sur vision joue un rôle privilégié dans une perspective systémique de la communication. Si le schéma de la figure 1 illustrant la complexité des combinatoires successives s'avérait juste, on pourrait reconstituer plusieurs scénarios possibles : 


\section{Scénario « rectilinéaire » alphanumérique}

14 Il privilégie résolument l'écrit, c'est le domaine de l'intellection pure, de l'échange fortement codé et sur codé. L'efficacité peut être très grande mais dans un domaine limité d'expression. La sur vision se manifeste essentiellement dans le domaine symbolique et les connotations.

\section{Scénario alphanumérique « mixte »}

15 Tout en recourant fortement à l'écrit, il laisse une place importante au non-verbal, essentiellement visuel (ne peut-on réinterpréter les lettrines et les enluminures de cette façon ou en termes plus modernes l'iconographie sémantique au service du texte). Avec les performances des meilleurs hypermédias, il va jusqu'à élargir l'apport visuel à la gestuelle. Que l'on songe à la " programmation affective " actuellement développée au MIT qui vise, entre autres, à permettre de déclencher des interactions entre un lecteur, un texte et un « assistant » cybernétique (par exemple le petit tigre « Tigrou ») en recourant au non-verbal pour faire découvrir/reconstruire le sens des textes et le goût de leur interprétation. Les sourires, les mimiques interactives induisent des interprétations ou une appétence pour des sens plus ou moins masqués - en retrouvant les recettes des précepteurs qui mettent en scène des textes même a priori rébarbatifs ${ }^{10}$ Dans des domaines autres que littéraires, des précurseurs tels que le prix Nobel de physique Richard Feynman ont montré que le non-verbal pouvait être sollicité en renforcement d'actes pédagogiques ${ }^{11}$

\section{Scénario audio-scripto-visuel interactif}

Outre le rappel toujours utile des recherches de Jean Cloutier ${ }^{12}$, ce scénario réhabiliterait une partie des "vieux» travaux sur l'audiovisuel éducatif. Pour y parvenir, il suffit de prendre pour point de départ des référents audiovisuels prégnants et de s'en servir comme tremplins ou amplificateurs (« boosters ») de sur vision et peutêtre un jour de « sur audition " pour accompagner la perception. Les exemples actuels viennent essentiellement du cinéma destiné aux jeunes (les "blockbusters») qui fonctionnent sur cette base en déclenchant des phénomènes distanciateurs de la perception classique ${ }^{13}$

\section{Scénario gestaltiste interactif.}

17 Un jour viendra où les meilleurs cours - qui ne présentent jamais seulement un strict « contenu » mais tout un environnement cognitif interactif (et affectif) typiquement mcluhanien ${ }^{14}$ - seront dispensés à l'aide d'«assistants pédagogiques" recourant simultanément au verbal codé et sur codé ainsi qu'à des inductions non-verbales aussi diversifiées qu'efficaces (mise en page interactive, gestuelle d'accompagnement ou de soulignement, bruitages et autres ambiances ou "paysages sonores", et plus tard pourquoi pas, d'autres canaux comme l'olfactif ou le tactile...). On pourra alors parler d'une extension de la sur vision à une sorte de « sur perception ». 


\section{Conclusion : une alliance entre sémantique et esthétique?}

18 l'efficacité pédagogique) et du schéma intégrateur de la complexité de l'information comme méta combinatoire, c'est-à-dire de combinatoires de niveaux inférieurs, nous a conduit à la proposition au cœur de cette communication : il est désormais possible de construire des systèmes informatiques interactifs capables de gérer du non-verbal inducteur de sens. Reste l'interrogation sur le caractère déterministe ou non de cet accompagnement. Va-t-on aboutir à une «ingénierie sociale " comme le disait sous forme de boutade Abraham Moles à Compiègne en 1978 au premier congrès Inforcom, ingénierie dans laquelle les systèmes détermineront totalement l'orientation de la perception et du sens reconnu ou recréé ?

Il nous semble qu'il n'en est rien, d'abord parce que la sur vision, prise comme exemple simple d'inducteur non-verbal de sens repose sur la «théorie distanciatrice ", laquelle est par nature non-déterministe, et ensuite parce que toute représentation systémique inclut un principe d'incertitude qui empêche toute velléité d'autoréférence «totalitaire ». C'est le moment de se souvenir du lien entre la perception sémantique et la perception esthétique. Outre son rôle de catalyseur, le non-verbal - pour nous la sur vision - remplit aussi une fonction esthétique, laquelle réfère non pas à une quelconque transmission linéaire ni même circulaire mais à une activation de matrices de sens, un peu comme Pierre Lévy le décrivait dans la Machine Univers ${ }^{15}$ - De la sorte, le non-verbal, en introduisant du bruit dans le processus, redonnerait de la liberté à Facteur individuel.

\section{NOTES}

1. L'identification n'est qu'un constituant de la triade "identification/projection/transfert » (IPT) qui permet de rendre compte de la multiplicité et de la diversité des profils individuels. Nous avons établi plusieurs fois que les individus peuvent être plutôt «identificateurs" (ils s'identifient avec la psychologie de leur modèle), ou "projectifs" (ils se projettent sur leur modèle en conservant leur propre psychologie). A ces deux catégories de base, il faut ajouter le transfert dans ses composantes psychologiques ou psychanalytiques.

2. Voir notamment notre ouvrage théorique : La distanciation. Essai sur la société médiatique, Paris, L'Harmattan. 1992.

3. Cf. le compte rendu de cette manifestation dans La Lettre d'inforcom $n^{\circ} 54$, automne 1998, p. 5.

4. Erwing Goffman, La Mise en scène de la vie quotidienne I. La présentation de soi. 2. Les relations en public. Paris. Éditions de Minuit, 1973. Voir aussi Les Rites d'interaction. Paris. Éditions de Minuit. 1974. ainsi que Jean-Luc Michel. La distanciation. Essai sur la société médiatique, op. cit., p. 85. sq.

5. À cet effet, nous avons aussi cherché à mettre au point le concept homologue de "suraudition » pour les phénomènes acoustiques, mais il s'agit $\mathrm{d}^{*}$ un domaine beaucoup plus complexe, ne serait ce que parce que les gestalts acoustiques sont difficiles à concevoir et décrire. Très peu d'auteurs se sont intéressés à cette question. Une des plus intéressantes contributions a été taire par 
Stephen McAdams et Emmanuel Bigand, Penser les sons. Psychologie cognitive de l'audition. Paris, P.U.F., 1994. Voir aussi le site web : http ://www.ircam.tr/departements/recherche/psycho.html

6. Revue Planète $\mathrm{n}^{\circ} 2,1961$. Cet auteur y parle de "survision ": "Tout était vu simultanément et de partout », p. 46. Pour une présentation détaillée de l'usage de la survision dans l'enseignement des mathématiques, on peut se reporter à notre article dans Communication et langages" S4, $2^{\mathrm{e}}$ trimestre 1990.

7. Quelques rares auteurs se sont intéressés à cet aspect des choses. On peut citer deux exemples très différents avec d'un côté les travaux d'Antoine de la Garanderie et sa « gestion mentale » et de l'autre un vieux livre de Jean-Jacques Henriot avec des conseils pratiques pour apprendre à se distaneier et "survoir» (même si cet auteur n'emploie pas ce tenue, l'idée y est exprimée). Antoine de la Garanderie, Comprendre et imaginer. Paris. Bayard, 1977, et Jean-Jacques Henriot, L'enfant, l'image et les médias. TV, publicité. BD. Paris, Vie et Santé-Horvath, 1986.

8. Sherry Turkle, The Second Self '[Les Enfants de l'ordinateur], Paris, Denoël, 1986.

9. Parmi les ouvrages d'André Leroi-Gourhan, voir tout particulièrement Le Geste et la parole, technique et langage. Paris, Albin Michel. 1964, ainsi que nos articles dans la revue de la SBS (Société de bibliologic et de schématisation). Références sur notre site web : http ://lepsie.univst-etienne.fr/inforconi/Notice_JLM.html

10. Pour une présentation détaillée, se reporter au site web du MIT: http:// www.media.mit.edu/affect/

11. Les tomes de ses cours de physique regorgent de schémas, anecdotes et facéties diverses qui induisent par du non-verbal graphique une appropriation et une mémorisation plus grandes des points les plus délicats à comprendre.

12. Une version web de son ouvrage fondamental de 1973 mais épuisé depuis longtemps Emerec, est disponible sur le site web que ce chercheur canadien a ouvert en 1999: http:// www.emerec.com

13. Naturellement, les tenants de réterne] retour philosophique peuvent soutenir qu'il ne s'agit que d'une "résurgence technomoderniste du monde des apparences platonicien ", mais ceci ne fait guère avancer la question qui nous occupe.

14. La recherche française est volontiers critique vis-à-vis de McLuhan. Sans entrer dans ce vieux débat, il nous semble que s'il est au moins une idée du « prophète de Toronto " qui n'a pas pris une ride, c'est bien son aphorisme provocateur dont on n'a pas encore épuisé le sens ni le génie ("The média is the message»).

15. Pierre Lévy, La Machine Univers, Paris, La Découverte, 1987.

\section{AUTEUR}

JEAN-LUC MICHEL

Université de Saint-Étienne 